12

\title{
Рост перегревных неустойчивостей в метастабильном металле
}

\author{
(C) В.И. Орешкин
}

Институт сильноточной электроники СО РАН, Томск, Россия

Томский политехнический университет, Томск, Россия

E-mail: oreshkin@ovpe.hcei.tsc.ru

Поступило в Редакцию 22 июня 2018 г.

На основе методов теории малых возмущений проанализировано развитие перегревных неустойчивостей в процессе электрического взрыва проводников. На начальной стадии электрического взрыва, после плавления металла, вещество может находиться в трех различных фазах: жидкость, двухфазная область (жидкость + пар), метастабильная жидкость (перегретый металл). Проведен сравнительный анализ роста перегревных неустойчивостей в зависимости от фазы, в которой может пребывать металл. Показано, что с точки зрения роста перегревных неустойчивостей наиболее нестабильной фазой оказывается метастабильная перегретая жидкость.

DOI: $10.21883 /$ PJTF.2018.20.46807.17432

Интерес к исследованию процессов, протекающих при электрическом взрыве проводников (ЭВП), определяется, с одной стороны, различными техническими приложениями [1-5], в том числе связанными с инерциальным термоядерным синтезом [6,7]. С другой стороны, ЭВП является важным источником информации об экстремальных состояниях вещества, так как при этом достигаются такие значения термодинамических параметров, которые невозможно получить в иных лабораторных экспериментах $[1,8]$. В процессе ЭВП, после плавления, фазовая траектория металла проходит вдоль кривой, называемой бинодалью, которая разделяет жидкость и в зависимости от состояния, в котором пребывает металл, либо двухфазную область (парокапельную смесь), либо метастабильную перегретую жидкость. В парокапельной смеси давление не зависит от плотности и в равновесии равно давлению насыщенных паров металла при данной температуре. Если же металл в это время находится в метастабильном состоянии - в виде перегретой растянутой жидкости, то давление может быть и отрицательным. На- 
против, в жидкой фазе в силу плохой сжимаемости жидкости давление быстро растет с ростом плотности. В процессе нагрева (при протекании тока) вещество расширяется и попадает в область с низким давлением, где магнитное давление превышает давление насыщенных паров (максимально возможное давление в двухфазной области), поэтому металл снова сжимается до жидкого состояния. В результате возникают звуковые колебания [1], период которых определяется отношением характерного продольного размера проводника к скорости звука [9], в проводниках микронных размеров он составляет величину порядка единиц наносекунд.

Как свидетельствуют многочисленные экспериментальные данные [10-15], ЭВП сопровождается появлением в проводнике страт. Существуют две наиболее вероятные причины появления страт. Первая - рост магнитогидродинамических неустойчивостей в виде перетяжек [16], вторая - рост перегревных неустойчивостей [17]. При развитии перетяжек решающую роль играет магнитное поле, создаваемое протекающим через проводник током, а структура перегревных неустойчивостей определяется характером зависимости удельного сопротивления вещества от его термодинамических параметров. Сравнение характерных времен развития этих неустойчивостей с временем взрыва проводника показывает, что перетяжки успевают развиться лишь при относительно низких плотностях тока, меньших $j \approx 10^{8} \mathrm{~A} / \mathrm{cm}^{2}$. Перегревные же неустойчивости развиваются всегда, так как их характерное время роста вне зависимости от геометрии проводника сравнимо с временем взрыва $[18,19]$.

Закономерности возникновения перегревных неустойчивостей рассматривались в работе [20], в которой на основе теории малых возмущений было получено дисперсионное уравнение, описывающее связь между мгновенным инкрементом нарастания $\gamma$ и аксиальной компонентой волнового вектора $k_{z}$, которое имело следующий вид:

$$
\gamma \approx \frac{j^{2}\left(\frac{\partial \delta}{\partial T}-\frac{\rho}{T^{*}} \frac{\partial \delta}{\partial \rho}\right)-k_{z}^{2} \kappa}{\rho c_{V}+\frac{p}{T^{*}}},
$$

где $\rho, T, p, c_{V}$ - плотность, температура, давление и теплоемкость вещества соответственно; $\kappa, \delta-$ коэффициент теплопроводности и

Письма в ЖТФ, 2018, том 44, вып. 20 
удельное сопротивление; $T^{*}-$ функция с размерностью температуры:

$$
T^{*}=\rho\left(\frac{\partial p}{\partial \rho}+\frac{\gamma^{2}}{k_{z}^{2}}\right)\left(\frac{\partial p}{\partial T}\right)^{-1} .
$$

Если в выражении (1) пренебречь тепловым давлением, то перегревные неустойчивости можно характеризовать $[18,20]$ максимальным инкрементом нарастания (при $k_{z} \rightarrow 0$ )

$$
\gamma_{m}=\frac{j^{2}}{\rho c_{V}} \frac{\partial \delta}{\partial T}
$$

и минимальной длиной волны (при $\gamma=0)$

$$
\lambda_{\min }=\frac{2 \pi}{j} \sqrt{\kappa\left(\frac{\partial \delta}{\partial T}\right)^{-1}} .
$$

Поскольку для большинства металлов в твердотельном и жидком состояниях производная сопротивления от температуры $\partial \delta / \partial T$ положительна, моды с длинами волн $\lambda>\lambda_{\min }$ нестабильны и их амплитуды растут экспоненциально; коротковолновые же моды (с длинами волн $\left.\lambda<\lambda_{\min }\right)$ затухают за счет теплопроводности.

В работе [20] обсуждались закономерности развития перегревных неустойчивостей для жидкости и парокапельной смеси, однако для метастабильной жидкости такие закономерности не рассматривались. В то же время эксперименты $[9,21]$ по электрическому взрыву фольг, целью которых являлось исследование распада метастабильного металла, показали, что при значениях вложенной в проводник энергии $0.3-0.5$ от энергии сублимации время распада перегретой жидкости превышает $100 \mathrm{~ns}$. Поэтому вероятно, что при относительно больших плотностях тока и как следствие при малых временах взрыва металл может попадать в метастабильное состояние. Основная цель настоящей работы - распространить анализ закономерностей роста перегревных неустойчивостей, проведенный в работе [20], на метастабильную фазу металла (перегретая жидкость).

Представим зависимость давления от плотности и температуры в следующем приближенном виде:

в жидкой и двухфазной областях [20]

$$
p=C \rho^{a} T^{b},
$$

Письма в ЖТФ, 2018, том 44, вып. 20 
а в метастабильной перегретой жидкости

$$
p=C\left[\left(\frac{\rho}{\rho_{x}}\right)^{a}-1\right] T^{b},
$$

где $\rho_{x}-$ некоторая кривая, являющаяся функцией температуры, на которой давление равняется нулю $(p=0)$, при $\rho<\rho_{x}$ давление становится отрицательным. При низких температурах положение кривой $\rho_{x}$ фактически совпадает с „жидкой“ бинодалью, а с ростом температуры и соответственно с ростом давления насыщенного пара эта кривая все более углубляется в метастабильную область.

Вычисления мгновенных инкрементов нарастания (значения показателей степени $a$ и $b$ ) основывались на широкодиапазонных полуэмпирических уравнениях состояния [22], в которых предусматривается наличие метастабильных состояний как перегретой жидкости, так и переохлажденного пара. Значения $a$ и $b$ для жидкости и парокапельной смеси приведены в [20], а для перегретого металла их значения равны $a \approx 10, b \approx-2.3$. Удельное сопротивление алюминия и его производные по температуре и плотности определялись по таблицам [23].

На рис. 1 для алюминиевого проводника представлены дисперсионные кривые, отражающие связь между мгновенным инкрементом нарастания $\gamma$ и аксиальной компонентой волнового вектора $k_{z}$ при плотности тока $j=10^{8} \mathrm{~A} / \mathrm{cm}^{2}$ и температуре $T=0.4 \mathrm{eV}$. Кривые рассчитаны при плотности вещества, близкой к плотности на бинодали $\rho_{b i n} \approx 1.6 \mathrm{~g} / \mathrm{cm}^{3}$, где возможно существование жидкости, парокапельной смеси и перегретого металла, и при давлении, равном по абсолютному значению давлению насыщенных паров $p= \pm 156 \mathrm{~atm}$ (в метастабильной области давление отрицательное). Сплошными линиями на рис. 1 показаны кривые, рассчитанные с помощью (1) при учете (2), (5) и (6), а штриховой линией - значения $\gamma_{m}$, вычисленные с помощью (3) без учета давления.

Поскольку при колебаниях возле бинодали металл попадает в жидкую фазу, в которой моды с длинами волн, меньшими $\lambda_{\min }$, затухают за счет теплопроводности (см. рис. 1), вероятно, что при ЭВП наиболее нестабильными будут моды с $\lambda \approx \lambda_{\text {min. }}$ При заданных значениях плотности тока и температуры величины $\lambda_{\min }$ и $\gamma_{m}$ являются функциями плотности вещества. Зависимости этих функций от плотности при $j=10^{8} \mathrm{~A} / \mathrm{cm}^{2}$ и $T=0.4 \mathrm{eV}$ представлены на рис. 2, $a$. Вертикальными

5 Письма в ЖТФ, 2018, том 44, вып. 20 


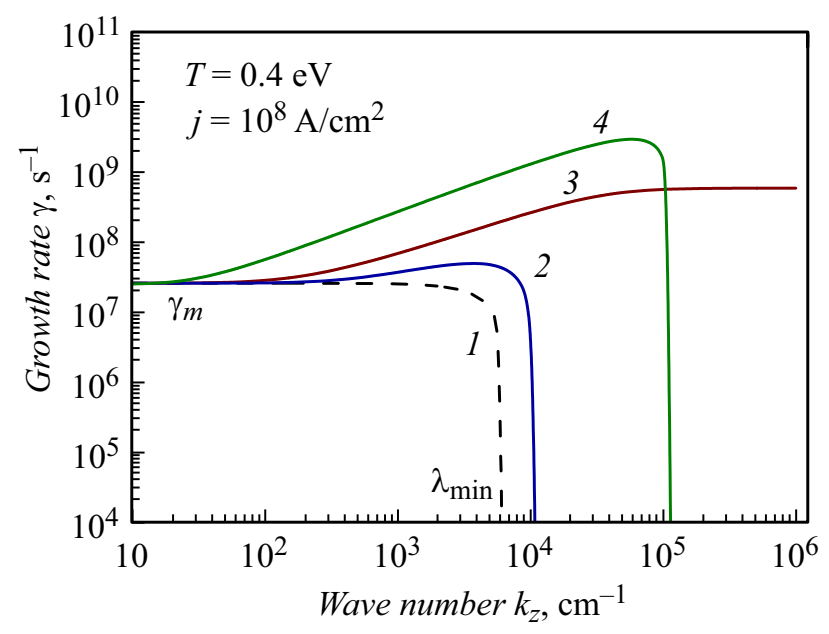

Рис. 1. Дисперсионные кривые, отражающие связь между мгновенным инкрементом нарастания $\gamma$ и аксиальной компонентой волнового вектора $k_{z}$. 1 - без учета давления, 2 - жидкая фаза, 3 - парокапельная смесь, 4 метастабильная перегретая жидкость.

штрихпунктирными линиями на этом рисунке показаны нормальная плотность вещества $\rho_{0}$, плотность на бинодали $\rho_{b i n}$, плотность на спинодали $\rho_{s p}$, т. е. на кривой, ограничивающей метастабильную область при малых плотностях вещества. На рис. 2, $b$ приведены зависимости от плотности мгновенных инкрементов нарастания $\gamma$, рассчитанные на длинах волн, равных $\lambda_{\min }$ (рис. $2, a$ ). Из рис. 1 и $2, b$ видно, что с точки зрения роста перегревных неустойчивостей наиболее нестабильной фазой является метастабильная перегретая жидкость.

Анализ результатов расчетов показывает, что на рост перегревных неустойчивостей основное влияние оказывают два дестабилизирующих фактора. Первый - наличие положительной производной от удельного сопротивления по температуре, т.е. $\partial \delta / \partial T>0$. Второй - наличие отрицательной производной от удельного сопротивления по плотности вещества, т.е. $\partial \delta / \partial \rho<0$. В длинноволновой части спектра значения мгновенных инкрементов нарастания определяются в основном производной $\partial \delta / \partial T$. В этой части спектра при близких условиях (при

Письма в ЖТФ, 2018, том 44, вып. 20 

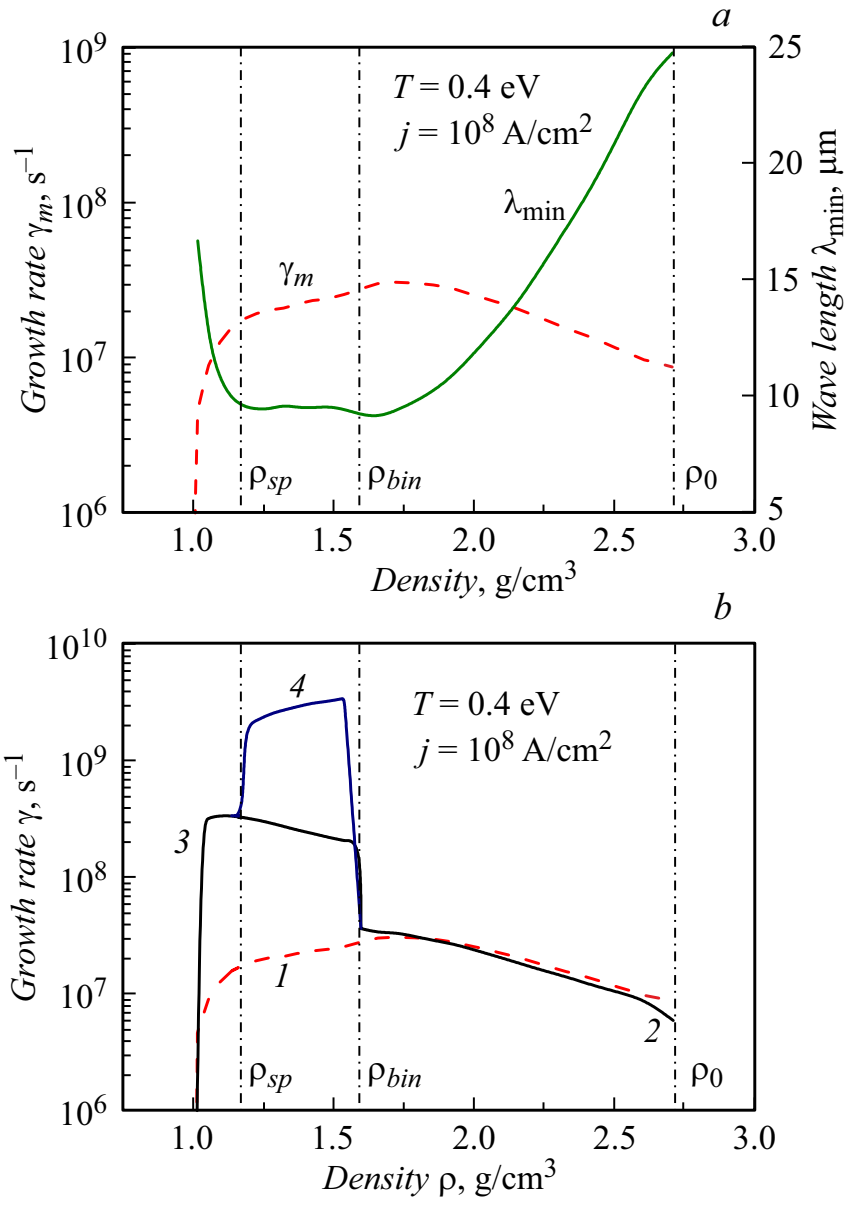

Рис. 2. Зависимости от плотности значений величин $\lambda_{\min }, \gamma_{m}(a)$ и инкрементов нарастания перегревных неустойчивостей $(b) .1-\gamma_{m}, 2-$ жидкая фаза, $3-$ парокапельная смесь, 4 - метастабильная перегретая жидкость.

приблизительно равных температурах и плотностях) значения $\gamma$ оказываются близки между собой для всех фазовых состояний вещества. В коротковолновой части спектра значения мгновенных инкрементов

$5^{*}$ Письма в ЖТФ, 2018, том 44, вып. 20 
нарастания определяются в основном производной $\partial \delta / \partial \rho$. В этой области спектра характер зависимости мгновенных инкрементов нарастания от волнового числа существенно различается в разных частях фазовой диаграммы.

Таким образом, расчеты показывают, что в двухфазной и метастабильной областях амплитудные значения $\gamma$ могут более чем на два порядка превышать максимальные значения инкрементов в жидкости. Это позволяет сделать вывод, что перегревные неустойчивости растут значительно быстрее, если в процессе электрического взрыва фазовая траектория металла попадает в двухфазную или метастабильную область.

Работа поддержана РФФИ (грант № 17-08-00459) и проектом Комплексной программы ФНИ СО РАН.

\section{Список литературы}

[1] Буриев В.А., Калинин Н.В., Лучинский А.В. Электрический взрыв проводников и его применение в электрофизических установках. М.: Энергоиздат, 1990. $288 \mathrm{c}$.

[2] Месяи Г.А. Импульсная энергетика и электроника. М.: Наука, 2004. 704 с.

[3] Lerner M.I., Glazkova E.A., Lozhkomoev A.S., Svarovskaya N.V., Bakina O.V., Pervikov A.V., Psakhie S.G. // Powder Technol. 2016. V. 295. P. 307-314.

[4] Кривочеев С.И., Титков В.В., Шнеерсон Г.А. // ЖТФ. 1997. Т. 67. В. 4. C. $32-47$.

[5] Суркаев А.Л. // ЖТФ. 2015. Т. 85. В. 7. С. 37-44.

[6] Awe T.J., Peterson K.J., Yu E.P., McBride R.D., Sinars D.B., Gomez M.R., Jennings C.A., Martin M.R., Rosenthal S.E., Schroen D.G., Sefkow A.B., Slutz S.A., Tomlinson K., Vesey R.A. // Phys. Rev. Lett. 2016. V. 116. N 6. P. 065001.

[7] Александров В.В., Гасилов В.А., Грабовский Е.В., Гричук А.Н., Лаухин Я.Н., Митрофанов К.Н., Олейник Г.М., Ольховская О.Г., Сасоров П.В., Смирнов В.П., Фролов И.Н., Шевелько А.П. // Физика плазмы. 2014. Т. 40. № 12. C. $1057-1073$.

[8] Exploding wires / Eds W.G. Chace, H.K. Moor. N. Y.: Plenum Press, 1959-1968. V. $1-4$.

[9] Oreshkin V.I., Zhigalin A.S., Rousskikh A.G., Kuznetsov V.V. // J. Eng. Thermophys. 2013. V. 22. N 4. P. 288-297.

Письма в ЖТФ, 2018, том 44, вып. 20 
[10] Sinars D.B., Shelkovenko T.A., Pikuz S.A., Romanova V.M., Chandler K.M., Greenly J.B., Hammer D.A., Kusse B.R. // Phys. Plasmas. 2000. V. 7. N 2. P. $429-432$.

[11] Sarkisov G.S., Struve K.W., McDaniel D.H. // Phys. Plasmas. 2004. V. 11. N 10. P. 4573-4581.

[12] Белько В.О., Емельянов О.А. // Письма в ЖТФ. 2009. Т. 35. В. 18. С. 58-64.

[13] Kaselouris E., Dimitriou V., Fitilis I., Skoulakis A., Koundourakis G., Clark E.L., Bakarezos M., Nikolos I.K., Papadogiannis N.A., Tatarakis M. // Nature Commun. 2017. V. 8. P. 1713.

[14] Baksht R.B., Rousskikh A.G., Zhigalin A.S., Oreshkin V.I., Artyomov A.P. // Phys. Plasmas. 2015. V. 22. N 10. P. 103521.

[15] Awe T.J., Yu E.P., Yates K.C., Yelton W.G., Bauer B.S., Hutchinson T.M., Fuelling S., Mckenzie B.B. // IEEE Transact. Plasma Sci. 2017. V. 45. N 4. Pt 1. P. 584-589.

[16] Абрамова К.Б., Златин Н.А., Перегуд Б.П. // ЖЭТФ. 1975. Т. 69. В. 6. C. 2007-2022.

[17] Валуев А.А., Дихтер И.Я., Зейгарник В.А. // ЖТФ. 1978. Т. 48. В. 10. C. 2088-2096.

[18] Орешкин В.И. // Письма в ЖТФ. 2009. Т. 35. В. 1. С. 76-82.

[19] Rousskikh A.G., Oreshkin V.I., Chaikovsky S.A., Labetskaya N.A., Shishlov A.V., Beilis I.I., Baksht R.B. // Phys. Plasma. 2008. V. 15. N 10. P. 102706.

[20] Oreshkin V.I. // Phys. Plasma. 2008. V. 15. N 9. P. 092103.

[21] Орешкин В.И., Жигалин А.С., Русских А.Г., Чайковский С.А., Бакшт Р.Б. // Изв. вузов. Физика. 2017. Т. 60. № 8. С. 123-130.

[22] Fortov V.E., Khishchenko K.V., Levashov P.R., Lomonosov I.V. // Nucl. Instrum. Meth. Phys. Res. A. 1998. V. 415. N 3. P. 604-608.

[23] Desjarlais M.P. // Contrib. Plasma Phys. 2001. V. 41. N 2-3. P. 267-270.

Письма в ЖТФ, 2018, том 44, вып. 20 